\title{
Millimeter-sized Graphene Quantum Hall Devices for Resistance Standards
}

\author{
Yanfei Yang ${ }^{1,2}$, Chieh-Wen Liu ${ }^{1}$, Chiashain Chuang ${ }^{1}$, Guangjun Cheng ${ }^{1}$, Angela R. Hight Walker ${ }^{1}$, David \\ B. Newell ${ }^{1}$, and Randolph E. Elmquist ${ }^{1}$ \\ ${ }^{1}$ National Institute of Standards and Technology (NIST), Gaithersburg, MD 20899-8171 \\ yanfei.yang@nist.gov \\ ${ }^{2}$ Joint Quantum Institute, University of Maryland, College Park, MD 20742 \\ ${ }^{3}$ Graduate Institute of Applied Physics, National Taiwan University, Taipei, Taiwan, 10617
}

\begin{abstract}
We present a repeatable process to produce millimeter-sized graphene device for quantum Hall resistance (QHR) standard. Our large-area, homogeneous monolayer graphene is grown on silicon carbide (SiC) substrate at high temperature by an optimized epitaxial method. Well-developed quantum Hall plateaus have been observed in devices fabricated from samples annealed at $1900{ }^{\circ} \mathrm{C}$ by a clean lithography process. Metrological accuracy of better than $5 \times 10^{-9}$ has been observed in a $27 \mathrm{~mm}^{2}$, octagonal device at a record current level of 0.72 $\mathrm{mA}$ for temperatures between $1.6 \mathrm{~K}$ and $3.1 \mathrm{~K}$.

Index Terms - Epitaxial, FTG, homogeneous graphene, millimeter-sized, quantum Hall effect, Raman microscopy, uniform carrier density.
\end{abstract}

\section{INTRODUCTION}

The quantum Hall effect (QHE) provides a universal, stable, and reproducible electrical resistance standard that is determined by two fundamental physical constants: Plank constant $h$ and elementary charge $e$. Before the discovery of graphene, the universality of QHE has been implemented in metal-oxide semiconductor field-effect transistors (MOSFET) and $\mathrm{GaAs}$ heterostructures. In these devices, realization of metrology accuracy at the level of few parts per billion (ppb) requires magnetic field on the order of $10 \mathrm{~T}$ and low temperature in the range of $0.5 \mathrm{~K}-1.5 \mathrm{~K}$, with the measurement current at the level of tens $\mu \mathrm{A}$ [1].

Graphene is a natural 2D electronic system with charge carriers mimicking the behavior of Dirac electrons [2]. This unique electronic property leads to an anomalous QHE with a sequence of QHR plateaus as $R_{\mathrm{H}}(i)=h / e^{2} 4(i+1 / 2)(\mathrm{i}=1,2, \ldots)$. The plateau with the filling factor of $v=4(i+1 / 2)=2$ is particularly robust and of great interest to metrology measurement. Recently, the quantization accuracy of few ppb has been reported in several graphene Hall bar devices [3]-[5]. In the exfoliated graphene device $(30 \mu \mathrm{m}$ wide), the measurement current for $\sim 5 \mathrm{ppb}$ is limited to $\sim 10 \mu \mathrm{A}$ at $18 \mathrm{~T}$ and $60 \mathrm{mK}$. In the epitaxial graphene Hall bar with similar width $(30 \mu \mathrm{m})$, ppb-level quantization has been demonstrated with a measurement current of $60 \mu \mathrm{A}$, in a magnetic field of $14 \mathrm{~T}$, and with temperature up to $15 \mathrm{~K}$. The larger Hall bar (100 $\mu \mathrm{m}$ wide) made from CVD graphene on SiC substrate maintains the accuracy of $1 \mathrm{ppb}$ with $500 \mu \mathrm{A}$ at $1.3 \mathrm{~K}$ and in a magnetic field of $8 \mathrm{~T}$. Although the quantization of Hall resistance has negligible dependence on the Hall bar size [6], larger samples can sustain a higher measurement current which ensures a large signal-to-noise ratio. Other key features to achieve high precision measurement include low contact resistance and homogeneous carrier density in the channel.

We demonstrate that high performance graphene devices can be repeatedly produced using homogeneous epitaxial graphene grown on $\mathrm{SiC}$ by an optimized method. The homogeneity is validated with AFM, optical microscopy, Raman microscopy, and magnetotransport. The QHE measurements on a very large octagonal graphene device (27 $\mathrm{mm}^{2}$ ) show metrological accuracy of better than $5 \times 10^{-9}$ at 9 $\mathrm{T}$ for temperatures between $1.6 \mathrm{~K}$ and $3.1 \mathrm{~K}$, with a measurement current of $0.72 \mathrm{~mA}$.

\section{EPITAXIAL GRAPHENE GROWTH}

Our samples are square substrates $(7.6 \mathrm{~mm} \times 7.6 \mathrm{~mm})$ diced from $4 \mathrm{H}-\mathrm{SiC}(0001)$ wafers that have very low nominal off-set angle relative to the basal plane. Graphene are grown at high temperature using the face-to-graphite (FTG) technique [7] in Ar background gas. The samples has been treated with forming gas $\left(4 \% \mathrm{H}_{2}, 96 \% \mathrm{Ar}\right)$ while heating the furnace to $1050{ }^{\circ} \mathrm{C}$ with 10 to 20 minutes dwell time. We optimize our process according to feedback from magnetotransport measurements and surface characterization including optical microscopy and atomic force microscopy.

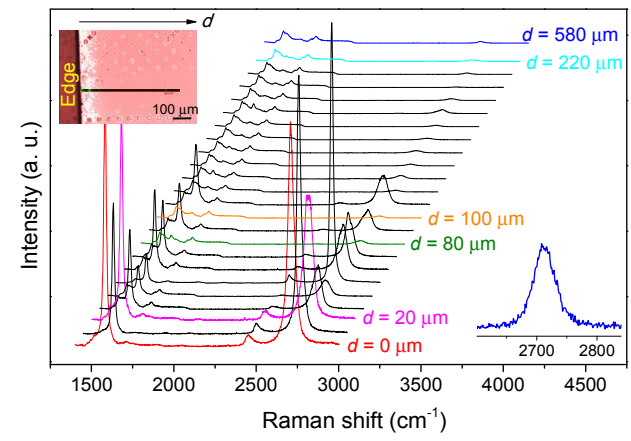

Fig. 1 Raman spectra of a line scan from the edge of a FTG sample $(d$ $=0)$ towards the center. Upper Inset: Optical image showing the line 
scan location (the black line). Lower Inset: Zoom-in region of $\mathrm{G}^{\prime}$ peak in the $d=580 \mu \mathrm{m}$ spectrum.

Raman microscopy confirms that thick graphene layers only occur near the sample edge. Figure 1 shows the Raman line spectra collected from a FTG sample processed at $1950{ }^{\circ} \mathrm{C}$ for 1800 s. Data were collected by scanning from the edge inward, along a line as shown in the upper inset of Fig. 1. The intensity of the graphitic Raman features, both $\mathrm{G}$ band $(\approx 1600$ $\left.\mathrm{cm}^{-1}\right)$ and the $\mathrm{G}^{\prime}$ band $\left(\approx 2700 \mathrm{~cm}^{-1}\right)$, attenuates rapidly with increasing distance from the sample edge. At interior distances $d>\approx 300 \mu \mathrm{m}$, the $\mathrm{G}^{\prime}$ peak takes on the shape of a single Lorentzian with a full width at half maximum of $\approx 40 \mathrm{~cm}^{-1}$, which is the fingerprint for monolayer graphene.

\section{MiLlimeteR-SIZED GRAPHENE QHE DEVICES}

We fabricated five millimeter-sized graphene devices from three FTG samples annealed at $1900{ }^{\circ} \mathrm{C}$. Fig. 2(a) shows the layout of three large Hall bars patterned on sample 1 (S1), which was annealed for 206 s. Fig. 2(b) shows the layout of the $27 \mathrm{~mm}^{2}$ octagonal devices fabricated on sample 2 (S2) and sample 3 (S3), which were annealed for $116 \mathrm{~s}$ and $210 \mathrm{~s}$, respectively. (a)

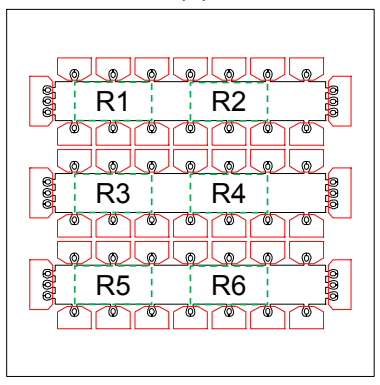

(c)

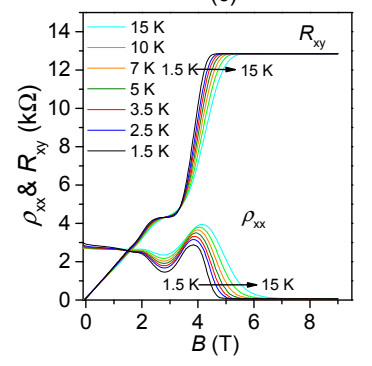

(b)

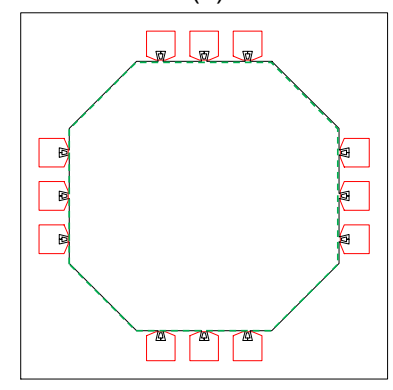

(d)
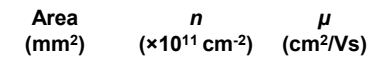

1.3 (S1_R1)

$1.3\left(\mathrm{~S} 11_{-} \mathrm{R} 2\right)$

$1.3\left(\mathrm{~S} 1{ }_{1} \mathrm{R} 3\right)$

1.3 (S1_R4)

1.3 (S1_R5)

$1.3\left(\mathrm{~S} 1 \_\mathrm{R} 6\right)$

27 (S2)

27 (S3)

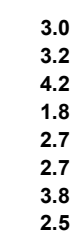

3.0
3.2
4.2
1.8
2.7
2.7
3.8
2.5

Figure 2. Layout and magnetotransport measurements of millimetersized graphene devices. (a) Layout of three large Hall bars $(0.8 \mathrm{~mm} \times$ $5.6 \mathrm{~mm}$ ) on S1. (b) Layout of the large octagonal device $(5.6 \mathrm{~mm}$ long and $5.6 \mathrm{~mm}$ wide) on S2 and S3. (c) Low temperature $\mathrm{AC}$ magnetotransport of S2 with measurement current of $1 \mu \mathrm{A}$. (d) Carrier density and mobility of S1-S3 at $T=1.5 \mathrm{~K}$

Well-developed $v=2$ QHR plateau has been observed in all five devices, similar to Fig. 2(c). The carrier density and mobility of the six regions in S1 (R1-R6) as well as S2 and S3 are listed in Fig. 2(d). The carrier density of S1 has a narrow distribution $n=(2.9 \pm 0.5) \times 10^{11} \mathrm{~cm}^{-2}$ associated with a high average mobility $\mu \approx 4200 \mathrm{~cm}^{2} / \mathrm{Vs}$. Metrology accuracy of better than $5 \mathrm{ppb}$ is measured in $\mathrm{S} 3$ at $9 \mathrm{~T}$ for temperatures between $1.6 \mathrm{~K}$ and $3.1 \mathrm{~K}$, with a current of $0.72 \mathrm{~mA}$.

\section{CONCLUSION}

We report a high-yield process to produce millimeter-sized graphene devices suitable for metrology measurements, with low contact resistance and uniform carrier density. This process includes a repeatable synthesis method to grow homogeneous graphene on $\mathrm{SiC}$, and clean fabrication techniques [8] for devices with low contact resistance and low carrier density. Our synthesis method is scalable to produce wafer-size, homogeneous graphene not only for metrology, but also desired by future electronics.

\section{ACKNOWLEDGEMENT}

The authors wish to thank Professor Paola Barbara of Georgetown University and Professor Chi-Te Liang of National Taiwan University for guidance support.

\section{REFERENCES}

[1] F. Delahaye and B. Jeckelmann, "Revised technical guidelines for reliable dc measurements of the quantized Hall resistance," Metrologia, vol. 40, pp. 217-223, 2003.

[2] K.S. Novoselov, A. K. Geim, S. V. Morozov, D. Jiang, M. I. Katsnelson, I. V. Grigorieva, S. V. Dubonos, and A. A., "Twodimensional gas of massless Dirac fermions in graphene," Nature, vol. 438, pp. 197-200, 2005.

[3] T. J. B. M. Janssen, J. M. Williams, N. E. Fletcher, R. Goebel, A. Tzalenchuk, R. Yakimova, S. Lara-Avila, S. Kubatkin, and V. Fal'ko, "Precisioni comparison of the quantum Hall effect in graphene and gallium arsenide," Metrologia, vol. 49, pp. 294306, 2012.

[4] M. Woszczyna, M. Friedemann, M. Götz, E. Pesel, K. Pierz, T. Weimann, and F. J. Ahlers, "Precision quantization of Hall resistance in transferred graphene," Appl. Phys. Lett., vol. 100, pp. 164106, 2012.

[5] R. Ribeiro-Palau, F. Lafont, J. Brun-Picard, D. Kazazis, A. Michon, F. Cheynis, O. Couturaud, C. Consejo, B. Jouault, W. Poirier, and F. Schopfer, "Quantum Hall resistance standard in graphene devices under relaxed experimental conditions," Nat. Nanotechnol., vol. 10, pp. 965-971, 2015.

[6] B. Jeckelmann and B. Jeanneret, "The quantum Hall effect as an electrical resistance standard," Rep. Prog. Phys., vol. 64, pp. 1603-1655, 2001.

[7] M. A. Real, E. A. Lass, F-H. Liu; T. Shen, G. R. Jones, J. A. SoonsD. B. Newell, A. V. Davydov, and R. E. Elmquist, "Graphene Epitaxial Growth on $\mathrm{SiC}(0001)$ for Resistance Standards," IEEE Trans. Instrum. Meas., vol. 62, pp. 14541460, 2013.

[8] Y. Yang, L.-I. Huang, Y. Fukuyama, F.-H. Liu, M. A. Real, P. Barbara, C.-T. Liang, D. B. Newell, R. E. Elmquist, "Low Carrier Density Epitaxial Graphene Devices On SiC," Small, vol. 11, pp. 90-95, 2015. 